\title{
Impact of chemotherapy cycles and intervals on outcomes of nonspinal Ewing sarcoma in adults: a real-world experience
}

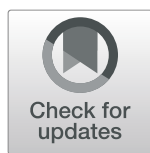

Jianjun Zhang ${ }^{1 *+} \mathbb{D}$, Yujing Huang ${ }^{1 \dagger}$, Yuanjue Sun ${ }^{2}$, Aina He ${ }^{1}$, Yan Zhou ${ }^{1}$, Haiyan Hu${ }^{1}$, Yang Yao ${ }^{1}$ and Zan Shen ${ }^{1 *}$

\begin{abstract}
Background: Adult Ewing sarcoma (ES) is a rare disease, the optimal treatment model is unknown. This study aimed to retrospectively analyze treatment-related prognostic factors of nonspinal ES in Chinese adults.

Methods: Eighty-one patients treated between January 2005 and December 2017 were included in the present study. Thirty-three (40.7\%) presented with metastatic disease at diagnosis. Eight patients were submitted to primary surgery followed by chemotherapy, while 73 patients received chemotherapy before and after surgery and/or local radiotherapy. The chemotherapy regimen included 8-17 cycles of vincristine, doxorubicin, and cyclophosphamide (VDC) alternating with ifosfamide and etoposide (IE) every 3 weeks. Clinical outcomes and safety were analyzed.

Results: VDC/IE chemotherapy was well tolerated in adult patients with ES. Multivariate Cox regression analyses revealed that chemotherapy of at least 12 cycles was a favorable independent prognostic factor of event-free survival (hazard ratio, $0.558 ; 95 \%$ confidence interval, $0.323-0.965 ; P=0.037$ ) and overall survival (hazard ratio, 0.424 ; $95 \%$ confidence interval, $0.240-0.748 ; P=0.003$ ). Similarly, a low frequency of chemotherapy delays was an independent prognostic factor of improved OS (hazard ratio, 0.438; 95\% confidence interval, 0.217-0.887; $P=0.022$ ).

Conclusion: Our study suggests that adults with ES should be treated with an aggressive multidisciplinary approach, intensive chemotherapy with adequate cycles and appropriate intervals can be recommended in this group.
\end{abstract}

Keywords: Ewing sarcoma, Chemotherapy, Adult, Outcome, Prognostic factor

\section{Background}

Ewing sarcoma (ES) is the second most common primary bone malignancy in children, but is extremely rare in adults [1-3]. The treatment of ES relies on a multidisciplinary approach that couples risk-adapted chemotherapy and local therapy (surgery, radiation therapy, or both). Chemotherapy plays a pivotal role in the treatment of ES. The regimen including vincristine, doxorubicin, and cyclophosphamide (VDC) alternating with ifosfamide and etoposide (IE) is a category 1 recommendation for ES in NCCN guideline [4]. However, there is no consensus on the optimal chemotherapy cycles and intervals. It was reported that VDC/IE every 3 weeks for 14 cycles contributed to similar survival compared with

\footnotetext{
* Correspondence: robustzhang168@aliyun.com; sshenzzan@vip.sina.com † Jianjun Zhang and Yujing Huang contributed equally to this work. ${ }^{1}$ Department of Oncology, Shanghai Jiao Tong University Affiliated Sixth People's Hospital, 600 Yishan Rd, Shanghai 200233, China Full list of author information is available at the end of the article
}

the same protocol for 17 cycles [5]. VDC/IE given at a 2week interval was found to be more effective than VDC/ IE given at a 3-week interval [5], but the NCCN panel only recommends this 2 -week compressed treatment in patients younger than 18 years.

As far as ES in adults is concerned, the optimal chemotherapy regimen remains unknown because current chemotherapy regimens are derived from clinical trials done in a predominantly pediatric population [5-9]. No prospective studies have been performed exclusively in adult ES because of the rarity of the diagnosis. It is not clear whether the pediatric regimens truly apply to adults [10]. The tolerability and efficacy of chemotherapy in older patients should be taken into account when extrapolating the protocols to adults $[3,11]$. On the other hand, previous clinical trials in ES were usually conducted in Europe and North America [2, 5-9, 12-14], and were rarely done in Asian countries. There are racial disparities 
in incidences of ES $[15,16]$, it is pending whether its response to treatment and prognosis also differ among races. In the present study, we retrospectively reviewed the data of adult ES treated by the same multidisciplinary team over 10 years in China. This study aimed to analyze chemotherapy-related factors that affected outcomes of this rare disease. To our knowledge, this is the largest study on adult ES from Asia. Primary ES of the spine was excluded in our cohort because it has special characteristics and prognostic factors [17].

\section{Methods}

\section{Patients}

This study was approved by the local ethics committee, informed consent was waived due to the retrospective nature of the study. Between January 2005 and December 2017, 87 adult patients with nonspinal ES were treated by the same multidisciplinary team in our center (Table 1). However, 6 patients were excluded either because of insufficient follow-up data or evidence of disease progression before completing first-line therapy. All 81 patients enrolled in the present study had histopathologically diagnosed ES, which met the diagnostic criteria described previously [17]. Evaluation for translocation t $(11 ; 22)$ (q24; q12) was not required to be enrolled in the study, it was performed in 39 patients. Initial staging procedures consisted of bone marrow biopsies and imaging studies. Imaging studies included X-rays, ultrasonic inspection, computed tomography and magnetic resonance imaging (MRI) of the primary tumor, a chest computed tomography scan, a bone scan or positron emission tomography.

\section{Treatments}

All patients received chemotherapy according to regimen described previously [17]. The regimen included vincristine $(1.4 \mathrm{mg} / \mathrm{m} 2$ on day 1 , maximum $2 \mathrm{mg})$, doxorubicin ( $75 \mathrm{mg} / \mathrm{m} 2$ on day 1$)$, and cyclophosphamide $(1.2 \mathrm{~g} / \mathrm{m} 2$ on day 1) (VDC) alternating with ifosfamide $(1.8 \mathrm{~g} / \mathrm{m} 2 /$ day, days $1-5)$ and etoposide $(100 \mathrm{mg} / \mathrm{m} 2 /$ day, days $1-5)$ (IE) every 3 weeks. When patients had received a cumulative doxorubicin dose of $300 \mathrm{mg} / \mathrm{m} 2$, dexrazoxane was administrated for cardioprotective purpose before each dose of doxorubicin at 10:1 dose ratio since 2012. Doxorubicin was replaced by actinomycin D $(1.25 \mathrm{mg} / \mathrm{m} 2)$ after reaching a cumulative dose of $450 \mathrm{mg} / \mathrm{m} 2$ or couldn't been tolerated by patients. Patients had a full blood test on the day prior to the cycle due date and chemotherapy was given if the neutrophil count was greater than $1.5 \times 10^{9} / \mathrm{L}$, platelet count greater than $80 \times 10^{9} / \mathrm{L}$ and biochemical parameters were within normal range. Otherwise, chemotherapy would be delayed until hematological recovery. As a routine, sustained grade 3-4 neutropenia for $>3$ days or neutropenic fever was an indication of dose reduction in the present study. Granulocyte colony-stimulating factor
Table 1 Demographic and Treatment Characteristics of patients

\begin{tabular}{|c|c|c|}
\hline Characteristics & Number & Percentage \\
\hline \multicolumn{3}{|l|}{ Gender } \\
\hline Male & 56 & 69.1 \\
\hline Female & 25 & 30.9 \\
\hline \multicolumn{3}{|l|}{ Age at diagnosis } \\
\hline$<30 y$ & 49 & 60.5 \\
\hline$\geq 30 y$ & 32 & 39.5 \\
\hline \multicolumn{3}{|l|}{ Primary site } \\
\hline Extremity & 25 & 30.9 \\
\hline Trunk & 56 & 69.1 \\
\hline \multicolumn{3}{|l|}{ Tumor origin } \\
\hline Skeletal & 57 & 70.4 \\
\hline Extraskeletal & 24 & 29.6 \\
\hline \multicolumn{3}{|l|}{ Stage } \\
\hline Localized & 48 & 59.3 \\
\hline Metastatic & 33 & 40.7 \\
\hline \multicolumn{3}{|l|}{ Diameter of primary tumor } \\
\hline$<8 \mathrm{~cm}$ & 50 & 61.7 \\
\hline$\geq 8 \mathrm{~cm}$ & 31 & 38.3 \\
\hline \multicolumn{3}{|l|}{ Local therapy } \\
\hline Surgery & 35 & 43.2 \\
\hline Radiotherapy & 18 & 22.2 \\
\hline Surgery + radiotherapy & 28 & 34.6 \\
\hline \multicolumn{3}{|c|}{ Number of chemotherapy cycles } \\
\hline$<12$ & 40 & 49.4 \\
\hline$\geq 12$ & 41 & 50.6 \\
\hline \multicolumn{3}{|c|}{ Frequency of chemotherapy delays } \\
\hline$<25 \%$ & 65 & 80.2 \\
\hline$\geq 25 \%$ & 16 & 19.8 \\
\hline \multicolumn{3}{|c|}{ Administration of dexrazoxane } \\
\hline YES & 39 & 48.1 \\
\hline NO & 42 & 51.9 \\
\hline
\end{tabular}

prophylaxis was recommended to avoid new episodes of neutropenia and delay of subsequent courses.

The timing and form of local therapy was at the discretion of the multidisciplinary treating team. Whenever possible, surgery was recommended for local treatment. If it was not feasible or not preferred by the patient, definitive radiotherapy would be performed. Indications for postoperative radiotherapy included gross residual disease and positive or close resection margins. The radiotherapy was delivered as previously described [17].

\section{Data collection}

A database was designed to retrospectively collect data on baseline demographic characteristics, treatment modalities, 
and clinical outcomes. Chemotherapy toxicity was reported according to the Common Terminology Criteria for Adverse Events version 4.0. Chemotherapy given $>3$ days later than the cycle due date was defined as a chemotherapy delay. Time to local therapy was defined as the time from the initiation of any treatment to local therapy. If a patient received both surgery and radiotherapy, the date of local therapy was defined by whichever occurred first. Event-free survival (EFS) was defined as the time from diagnosis to disease recurrence, progression, second malignancy, death from any cause, or last contact. Overall survival (OS) was defined as the interval between diagnosis and death from any cause or the most recent follow-up. Clinical responses were classified as complete response (CR), partial response (PR), stable disease (SD), and progressive disease (PD) according to response evaluation criteria in solid tumors for the soft-tissue component of the primary lesion as well as non-osseous metastases [18]. Missing data were collected from patients or their family members by telephone. Information regarding treatment at recurrence was not collected.

\section{Statistical analysis}

All analyses were performed using Statistical Package for the Social Sciences (SPSS), version 18.0 (Chicago, Illinois, USA). Continuous variables were presented as mean \pm SD. Qualitative variables were shown as absolute and relative frequencies. The $x^{2}$ or Fisher's exact test was used to compare proportions. Kaplan-Meier survival curves were used to estimate proportion surviving and the log-rank test was used to compare differences among subgroups. Cox regression models were used to identify independent prognostic factors. If variables were significant at the 0.1 level on univariate analysis, then they were included in the multiple regression. A $P$ value < 0.05 was considered significant.

\section{Results}

\section{Patient characteristics}

This study consisted of 81 patients (56 males, $25 \mathrm{fe}$ males), with a mean age of $31.0 \pm 9.4$ years (range 18-63 years) at diagnosis. Forty-nine patients $(60.5 \%)$ were $<$ 30 years of age, whereas 32 patients (39.5\%) were $\geq 30$ years. The primary tumors were skeletal in 57 (70.4\%) patients, while 24 (29.6\%) had extraskeletal disease. Twenty-five patients had extremity-based tumors, including those in the lower $(n=17)$ and upper $(n=8)$ extremities. 56 patients had trunk-based tumors, including those in the pelvic girdle $(n=23)$, in the thorax $(n=17)$, at head and neck $(n=9)$, and in abdomen and hip ( $n=$ 7). The diameter of primary lesion was $<8 \mathrm{~cm}$ in 50 cases and $\geq 8 \mathrm{~cm}$ in 31 patients. Among all 81 patients, $48(59.3 \%)$ patients presented with localized disease, 33 (40.7\%) patients presented with metastatic disease (14 with lung metastases, 10 with bone metastases, 1 with lymphonode metastases, 7 with both lung and bone metastases, 1 with simultaneous bone and bone marrow metastases) at diagnosis (Table 1).

All patients received chemotherapy, 8 after local treatment and 73 as neoadjuvant and adjuvant therapies. The median number of cycles received per patient was 12 (range, 8 to 17). For the 8 patients who received chemotherapy after local treatment, primary surgery was performed for curative purpose because the treating surgeon did not suspect the presence of ES. Among the remaining 73 patients, 27 patients underwent surgery of the primary tumors as local therapy, 18 patients received radiotherapy alone, and 28 patients received both. Overall, the median time to local therapy was 3.2 months (range, 0-7 months).

\section{Clinical outcomes}

The clinical response in all 73 patients who underwent neoadjuvant chemotherapy included 19 CRs, 37 PRs and 17 SDs, with an overall response rate of $76.7 \%$. Pathologic evaluation of chemotherapy-related tumor necrosis was available for 45 patients who received neoadjuvant chemotherapy; good response (necrosis of $\geq 90 \%$ of the resected specimen) was observed in $20 / 32(62.5 \%)$ patients with skeletal tumor and in 6/13 (46.2\%) patients with extraskeletal disease.

The median follow-up in this cohort was 36 months (range, 11-120 months). Local relapses occurred in 23 patients, 7 of them had undergone exclusive surgery as local therapy, 6 of them had received radiation as local therapy, and the other 8 patients had received combined surgery and radiotherapy, the incidences of local relapses in these three subgroups were similar $(P>0.05)$. Among the 23 patients with local relapses, 10 presented simultaneous metastatic relapses (including metastatic progression) and 13 delayed metastatic relapses. Thirty patients experienced isolated metastatic relapse as a first oncological event. Local and metastatic relapses occurred within a median interval of 23 months (range, 11-49 months). Overall, the 5year EFS was $22.0 \%$. Fifty-two patients died of disease progression, no death from any other cause occurred, leading to a 5 -year OS of $33.0 \%$.

\section{Safety}

Drug toxicities to the hematologic system, liver, kidneys, heart, bladder, mucosa and peripheral nerve were observed during chemotherapy. Grade 3-4 neutropenia was reported in 77 patients (95.1\%), grade 3-4 thrombocytopenia in 45 patients (55.6\%). Red blood cells or platelets transfusions were needed in $13(16.0 \%)$ and 9 (11.1\%) patients, respectively. Peripheral neurologic toxicity occurred in 10 patients; one patient discontinued vincristine early due 
to grade 3 neurologic toxicity. Toxicity-related dose reduction occurred in 48 patients (59.3\%) (Table 2). Forty-two patients $(51.8 \%)$ had at least one chemotherapy delay, while 16 patients had chemotherapy delays in more than $25 \%$ cycles. The major cause of chemotherapy delays was chemotherapy toxicity (87.9\%), other reasons accounted for $12.1 \%$. Notably, frequencies of dose reduction were similar in the two patient subgroups who received chemotherapy more or less than 12 cycles $(65.9 \%$ vs. $52.5 \%, P>0.05)$, so the number of chemotherapy cycles positively correlated with the total chemotherapy doses and the frequencies of

Table 2 Chemotherapy toxicity in patients

\begin{tabular}{|c|c|c|}
\hline Toxicity and grade & Number & Percentage \\
\hline \multicolumn{3}{|c|}{ Hematological toxicities } \\
\hline \multicolumn{3}{|l|}{ Neutropenia } \\
\hline All & 81 & 100 \\
\hline $3-4$ & 77 & 95.1 \\
\hline \multicolumn{3}{|l|}{ Anemia } \\
\hline All & 29 & 35.8 \\
\hline $3-4$ & 17 & 21.0 \\
\hline \multicolumn{3}{|c|}{ Thrombocytopenia } \\
\hline All & 76 & 93.8 \\
\hline $3-4$ & 45 & 55.6 \\
\hline \multicolumn{3}{|c|}{ Nausea and vomiting } \\
\hline All & 61 & 75.3 \\
\hline $3-4$ & 20 & 24.7 \\
\hline \multicolumn{3}{|l|}{ Hepatic dysfunction } \\
\hline All & 13 & 16.0 \\
\hline $3-4$ & 6 & 7.4 \\
\hline \multicolumn{3}{|l|}{ Renal dysfunction } \\
\hline $1-2$ & 4 & 4.9 \\
\hline \multicolumn{3}{|l|}{ Mucositis } \\
\hline All & 16 & 19.8 \\
\hline $3-4$ & 9 & 11.1 \\
\hline \multicolumn{3}{|l|}{ Cardiac toxicities } \\
\hline \multicolumn{3}{|l|}{ Arrhythmia } \\
\hline $1-2$ & 11 & 13.6 \\
\hline \multicolumn{3}{|c|}{ Myocardial ischemia } \\
\hline $1-2$ & 7 & 8.6 \\
\hline \multicolumn{3}{|l|}{ Hemorrhagic cystitis } \\
\hline $1-2$ & 4 & 4.9 \\
\hline \multicolumn{3}{|l|}{ Neurologic toxicity } \\
\hline All & 10 & 12.3 \\
\hline $3-4$ & 1 & 1.23 \\
\hline \multicolumn{3}{|l|}{ Dose reduction } \\
\hline All & 48 & 59.3 \\
\hline
\end{tabular}

chemotherapy delays positively correlated with chemotherapy intervals.

\section{Analysis of prognostic factors of survival}

Univariable analysis showed that stage $(P=0.071)$ and number of chemotherapy cycles $(P=0.058)$ showed a trend toward significance for EFS. These two factors were submitted to multivariable analysis. The results showed that both localized disease at diagnosis $(P=$ $0.045)$ and chemotherapy of at least 12 cycles $(P=0.037)$ were favorable independent prognostic factors of EFS (Table 3). As far as OS was concerned, univariable analysis showed that stage $(P=0.004)$ and number of chemotherapy cycles $(P=0.015)$ were significantly associated with OS. These two factors along with frequency of chemotherapy delays $(P=0.076)$ were submitted to multivariable analysis. Multivariable analysis showed that all these three factors were significant predictors of OS (Table 4). Chemotherapy of at least 12 cycles was associated with both improved EFS and OS in the present study (Fig. 1). Stratified analyses indicated that frequency of chemotherapy delays didn't affect EFS significantly (log-rank test, $P=0.364$ ). However, a low frequency of chemotherapy delays $(<25 \%)$ was a favorable independent predictor of OS in our patients $(P=0.022)$.

\section{Discussion}

At present, no standard treatment model has established for adult ES and the management is often institutionspecific [19-22]. Even for the most commonly used VDC/ IE chemotherapy regimen, the number of cycles ranges from 6 to 17 in different studies [5, 22, 23]. The optimal chemotherapy regimen for adult ES is controversial [10]. Tao et al. reported that there were no significant differences between anthracycline and platinum based chemotherapy regarding EFS and OS [19]. In contrast, Casey et al. and Ahmed et al. reported that treatment according to the pediatric protocol was significantly associated with improved survival $[21,24]$. In the present cohort, we also followed the pediatric VDC/IE protocol, unfortunately 50 patients didn't completed the planned 17 chemotherapy cycles due to various reasons: the most common reason was lack of money (21 patients, $42 \%$ ), followed by the belief that adults inevitably fare worse than children and increasing chemotherapy duration may not lead to improvement in survival (16 patients, 32\%), toxicity-related treatment abandonment occurred in eight patients (16\%), and other reasons accounted for five patients (10\%). Overall, VDC/IE chemotherapy was well tolerated in our patients. Our study provided real-world data in adult ES from Asian developing countries, and the aforementioned situation provided an opportunity for comparative analysis between different chemotherapy cycles and intervals. 
Table 3 Univariable and multivariable Cox proportional hazard regression analyses of event-free survival

\begin{tabular}{|c|c|c|c|c|}
\hline \multirow[t]{2}{*}{ Factor } & Univariable analysis & $P$ & Multivariable analysis & $P$ \\
\hline & \multicolumn{2}{|l|}{$\mathrm{HR}(95 \% \mathrm{Cl})$} & \multicolumn{2}{|l|}{ HR $(95 \% \mathrm{Cl})$} \\
\hline \multicolumn{5}{|l|}{ Gender } \\
\hline Male & Reference & & & \\
\hline Female & $1.107(0.614-1.993)$ & 0.735 & & \\
\hline \multicolumn{5}{|l|}{ Age at diagnosis } \\
\hline$<30 y$ & Reference & & & \\
\hline$\geq 30 y$ & $1.410(0.820-2.426)$ & 0.215 & & \\
\hline \multicolumn{5}{|l|}{ Primary site } \\
\hline Extremity & Reference & & & \\
\hline Trunk & $0.874(0.500-1.526)$ & 0.635 & & \\
\hline \multicolumn{5}{|l|}{ Tumor origin } \\
\hline Skeletal & Reference & & & \\
\hline Extraskeletal & $0.771(0.418-1.421)$ & 0.404 & & \\
\hline \multicolumn{5}{|l|}{ Stage } \\
\hline Localized & Reference & & Reference & \\
\hline Metastatic & $1.644(0.958-2.282)$ & 0.071 & $1.743(1.012-3.002)$ & 0.045 \\
\hline \multicolumn{5}{|l|}{ Diameter of primary tumor } \\
\hline$<8 \mathrm{~cm}$ & Reference & & & \\
\hline$\geq 8 \mathrm{~cm}$ & $0.800(0.458-1.398)$ & 0.434 & & \\
\hline \multicolumn{5}{|l|}{ Local therapy } \\
\hline Surgery & Reference & & & \\
\hline Radiotherapy & $1.504(0.733-3.087)$ & 0.266 & & \\
\hline Surgery + radiotherapy & $1.494(0.806-2.771)$ & 0.202 & & \\
\hline Time to local therapy & $0.899(0.732-1.104)$ & 0.309 & & \\
\hline \multicolumn{5}{|c|}{ Number of chemotherapy cycles } \\
\hline$<12$ & Reference & & Reference & 0.037 \\
\hline$\geq 12$ & $0.592(0.344-1.017)$ & 0.058 & $0.558(0.323-0.965)$ & \\
\hline \multicolumn{5}{|c|}{ Frequency of chemotherapy delays } \\
\hline$\geq 25 \%$ & Reference & & & \\
\hline$<25 \%$ & $0.739(0.380-1.439)$ & 0.374 & & \\
\hline \multicolumn{5}{|c|}{ Grade 3-4 chemotherapy toxicity } \\
\hline YES & Reference & & & \\
\hline NO & $0.382(0.053-2.778)$ & 0.342 & & \\
\hline \multicolumn{5}{|c|}{ Administration of dexrazoxane } \\
\hline YES & Reference & & & \\
\hline NO & $1.057(0.616-1.814)$ & 0.840 & & \\
\hline
\end{tabular}

HR Hazard ratio, $\mathrm{Cl}$ Confidence interval

It was reported that adult patients with ES had a worse prognosis compared to pediatric patients [5, 9, 23, 25-27]. As far as nonmetastatic ES was concerned, Granowetter et al. reported that the 5-year EFS was $77.5 \%$ for patients age 1 to 9 years, $69.4 \%$ for patients age 10 to 17 years, and $63.2 \%$ for patients older than 18 years [9]. Womer et al. reported that the 5-year EFS was 72 and $47 \%$ for patients younger and older than 18 years, respectively [5]. In the present study, we also observed a poor prognosis for adult ES, with a 5 -year OS of $33.0 \%$ and EFS of only $22.0 \%$. The reasons behind the poor prognosis for adult ES are unclear. Generally, the prognostic factors of ES include site of primary disease, tumor volume, response to chemotherapy, and presence of metastatic disease at diagnosis $[12,13,28]$. It is not clear if these findings from pediatric studies also apply to adults because several case series suggested that ES 
Table 4 Univariable and multivariable Cox proportional hazard regression analyses of overall survival

\begin{tabular}{|c|c|c|c|c|c|}
\hline \multirow[t]{2}{*}{ Factor } & Univariable analysis & \multirow[t]{2}{*}{$P$} & Multivariable analysis & \multirow[t]{2}{*}{$P$} & \\
\hline & $\mathrm{HR}(95 \% \mathrm{Cl})$ & & \multicolumn{2}{|l|}{ HR (95\% Cl) } & \\
\hline \multicolumn{6}{|l|}{ Gender } \\
\hline Male & Reference & & & & \\
\hline Female & $1.376(0.766-2.472)$ & 0.286 & & & \\
\hline \multicolumn{6}{|l|}{ Age at diagnosis } \\
\hline$<30 y$ & Reference & & & & \\
\hline$\geq 30 y$ & $1.450(0.839-2.507)$ & 0.183 & & & \\
\hline \multicolumn{6}{|l|}{ Primary site } \\
\hline Extremity & Reference & & & & \\
\hline Trunk & $1.105(0.620-1.971)$ & 0.735 & & & \\
\hline \multicolumn{6}{|l|}{ Tumor origin } \\
\hline Skeletal & Reference & & & & \\
\hline Extraskeletal & $0.660(0.346-1.261)$ & 0.209 & & & \\
\hline \multicolumn{6}{|l|}{ Stage } \\
\hline Localized & Reference & & & Reference & \\
\hline Metastatic & $2.269(1.294-3.976)$ & 0.004 & & $2.488(1.413-4.383)$ & 0.002 \\
\hline \multicolumn{6}{|l|}{ Diameter of primary tumor } \\
\hline$<8 \mathrm{~cm}$ & Reference & & & & \\
\hline$\geq 8 \mathrm{~cm}$ & $0.765(0.434-1.351)$ & 0.356 & & & \\
\hline \multicolumn{6}{|l|}{ Local therapy } \\
\hline Surgery & Reference & & & & \\
\hline Radiotherapy & $1.327(0.654-2.692)$ & 0.432 & & & \\
\hline Surgery + radiotherapy & $1.271(0.679-2.379)$ & 0.453 & & & \\
\hline Time to local therapy & $0.901(0.733-1.107)$ & 0.329 & & & \\
\hline \multicolumn{6}{|c|}{ Number of chemotherapy cycles } \\
\hline$<12$ & Reference & & & Reference & 0.003 \\
\hline$\geq 12$ & $0.505(0.291-0.874)$ & 0.015 & & $0.424(0.240-0.748)$ & \\
\hline \multicolumn{6}{|c|}{ Frequency of chemotherapy delays } \\
\hline$\geq 25 \%$ & Reference & & & Reference & \\
\hline$<25 \%$ & $0.537(0.270-1.067)$ & 0.076 & & $0.438(0.217-0.887)$ & 0.022 \\
\hline \multicolumn{6}{|c|}{ Grade 3-4 chemotherapy toxicity } \\
\hline YES & Reference & 0.610 & & & \\
\hline NO & $0.595(0.081-4.359)$ & & & & \\
\hline \multicolumn{6}{|c|}{ Administration of dexrazoxane } \\
\hline YES & Reference & & & & \\
\hline NO & $0.938(0.540-1.628)$ & 0.820 & & & \\
\hline
\end{tabular}

HR Hazard ratio, $\mathrm{Cl}$ Confidence interval

in adult populations was a more biologically aggressive variant of the disease [3, 27]. However, there is a uniform consensus that patients presenting with metastatic disease have an extremely poor prognosis, regardless of age. In our cohort, $40.7 \%$ of patients presented with metastatic disease at diagnosis, slightly more compared with other series [24, 29, 30], this may partially justify the dismal survival. When patients with metastatic disease at presentation were excluded, the 5-year OS and EFS increased to 44.0 and 31\% for the remaining 48 patients in the present study. Notsurprisingly, this outcome was worse than that of aforementioned pediatric patients with localized ES [5, 9]. However, these figures were still fewer than those from other recent adult cohorts: OS of $66 \%$ and EFS of $44.0 \%$ in the Casey et al. series of 76 cases [24], OS of $66 \%$ and EFS of $43.0 \%$ in the Fizazi et al. series of 129 cases [30]. 

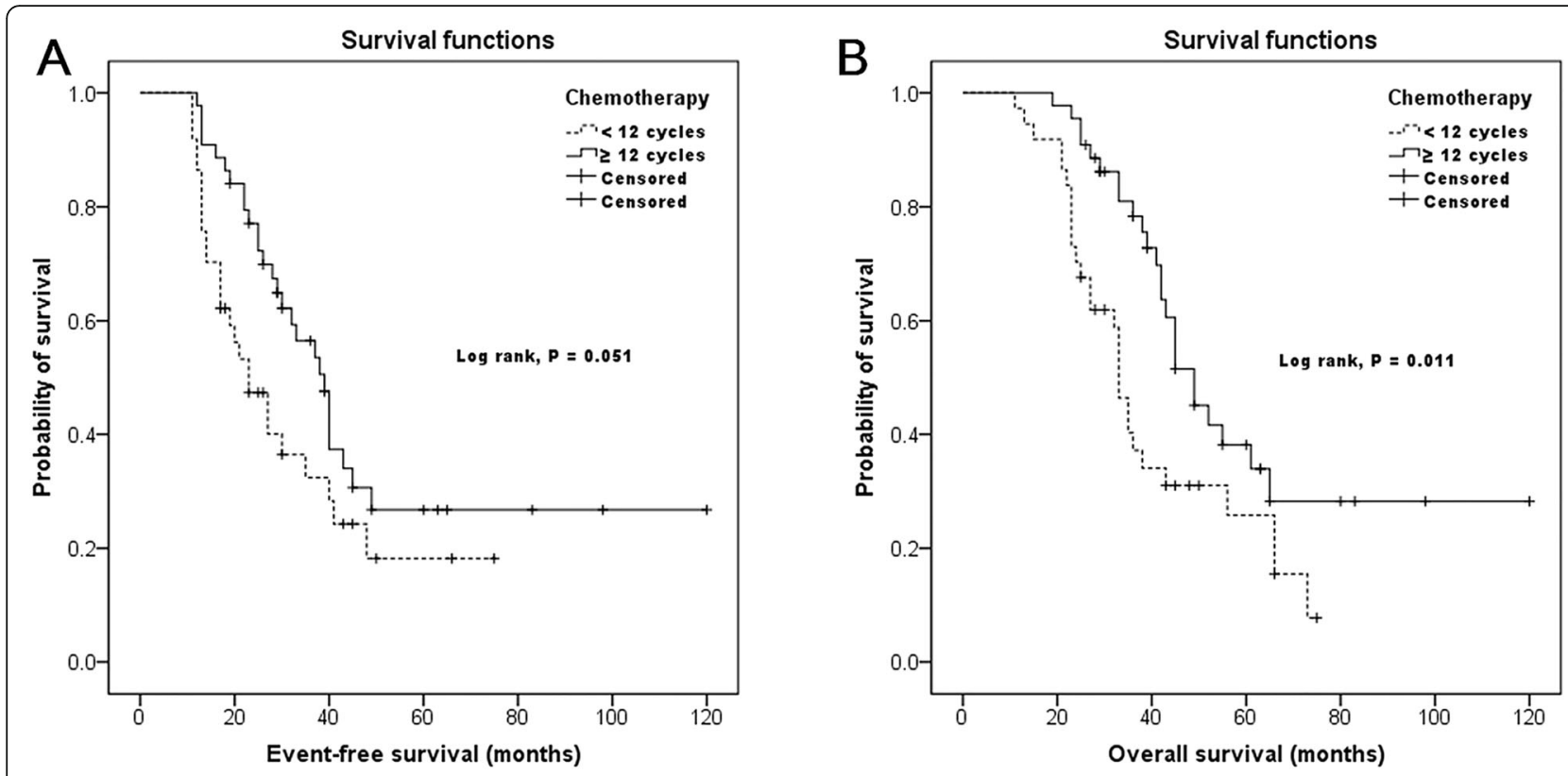

Fig. 1 Kaplan-Meier survival curves for event-free survival (a) and overall survival (b) according to number of chemotherapy cycles

To identify the factors that potentially affect the outcomes of our cohort, we conducted exploratory univariable and multivariable analyses. Interestingly, besides metastatic disease at diagnosis, chemotherapy of less than 12 cycles was also detrimental to both OS and EFS of our patients. Moreover, a high frequency of chemotherapy delays ( $\geq 25 \%$ ) was an unfavorable independent predictor of OS. As mentioned above, the number of chemotherapy cycles positively correlated with the chemotherapy doses and the frequencies of chemotherapy delays positively correlated with chemotherapy intervals. These findings suggested that the poor prognosis in the present study was partially due to insufficient chemotherapy doses and prolonged dose intervals. Dose-dense chemotherapy with shortening intervals showed an increase in survival of ES [5], whereas the benefit of dose escalation (increasing the dose of chemotherapy agents) studies has been less consistent and may be accompanied by other dose-limiting toxicities [31]. It was reported that lower doses of alkylating agents were detrimental to survival of ES [23], however, dose escalation of alkylating agents in the VDC/ IE regimen did not improve the outcome for patients with localized disease [9]. Based on our results, we strongly recommend aggressive treatment for adult ES to maintain adequate chemotherapy doses and appropriate intervals, with the help of supportive treatment. None of the other factors such as extremity/axial primary site, skeletal/extraskeletal tumor origin, diameter of primary tumor, local therapy modality and time to local therapy achieved statistical significance, but age older than 30 years at diagnosis (HR 1.45, $P=0.183$ ) showed a trend toward significance for OS. Although chemotherapy cycles and intervals can be affected by chemotherapy toxicity, the univariable analysis indicated that grade 3-4 chemotherapy toxicity didn't affect the EFS and OS of our patients significantly.

To further explore the potential racial disparity in survival of adult ES, we reviewed the outcomes of localized disease treated with similar cycles of VDC/IE regimen (Table 5).

Table 5 Clinical outcome of adults with localized Ewing sarcoma

\begin{tabular}{llll}
\hline References & Study group & CT regimens & Outcomes \\
\hline Womer et al. [5] & Prospective study & VDC/IE (including every 2-week protocol) & 14 cycles \\
& 31 patients $\geq 18$ y (2001-2005, USA) & VDC/IE & 3 -y EFS 43\% \\
Gupta et al. [23] & Retrospective study & 10 cycles & $3-y$ OS 59\% \\
& 24 patients $\geq 18$ y (1990-2005, Canada) & VDC/IE & $5-y$ OS 64\% \\
Seker et al. [22] & Retrospective study & 17 cycles & $3-y$ EFS 60\% \\
& 21 patients $\geq 19$ y (2000-2012, Turkey) & VDC/IE & $3-y$ OS 81\% \\
The present study & Retrospective study & 212 cycles & $5-y$ EFS 38\% \\
& (2005-2017, China) & & $5-y$ OS 51\% \\
& & &
\end{tabular}


The prognosis of our patients was better than that of Canadian series, while it was a little worse than the prognosis of cohorts from the USA and Turkey. The results showed no evidence of ethnical disparity in survival of adult ES. Further head-to-head comparative studies are needed to clarify this question.

Patients who receive high doses of anthracyclines are at risk for cardiotoxicity, dexrazoxane is often used as a cardioprotectant when prior doxorubicin reached a cumulative dose of $300 \mathrm{mg} / \mathrm{m}^{2}$ [32]. But controversy exists if dexrazoxane reduces the antitumor effect of doxorubicin and increases the risk of second primary malignancies [33]. In the present study, we found that there were no differences of EFS and OS in patients with or without administration of dexrazoxane (log-rank test, $P>0.05$ ), and there was no second primary malignancy occurred during the follow-up period. Our results were consistent with the findings from other recent large populationbased studies [32, 34, 35].

This study has several limitations. First, it was a retrospective, single-institution study with a small sample size. As a consequence, it was difficult to draw conclusions on all factors influencing outcomes. Second, we excluded patients for whom there was incomplete clinical data or evidence of disease progression before completing first-line therapy. This could have resulted in selection bias. To assess accurately the outcomes and prognostic factors of adult ES, large prospective clinical trials are needed.

\section{Conclusion}

The present study shows that VDC/IE chemotherapy is well tolerated in adult patients with ES, chemotherapy of at least 12 cycles is associated with better EFS and OS compared with less cycles of chemotherapy. Similarly, a low frequency of chemotherapy delays is an independent prognostic factor of improved OS. Our findings suggest that adults with ES should be treated with an aggressive multidisciplinary approach, intensive chemotherapy with adequate doses and appropriate intervals can be recommended in this group when the toxicity was tolerable.

\footnotetext{
Abbreviations

CR: Complete response; EFS: Event-free survival; ES: Ewing sarcoma; HR: Hazard ratio; IE: Ifosfamide and etoposide; MRI: Magnetic resonance imaging; NCCN: The National Comprehensive Cancer Network; OS: Overall survival; PD: Progressive disease; PR: Partial response; SD: Stable disease; SPSS: Statistical Package for the Social Sciences; VDC: Vincristine, doxorubicin, and cyclophosphamide
}

\section{Acknowledgements}

None.

\section{Authors' contributions}

$J Z$, and ZS conceived and designed the study, wrote, reviewed and edited the manuscript. YH, YY, HH and $Y Z$ performed the methodological development and analysis of the data. YS, YH and $\mathrm{AH}$ collected the data. All authors read and approved the final manuscript.

\section{Funding}

This work was supported by grants from the National Natural Science Foundation of China (No. 81503396), the National Key Research Project of Science and Technology Ministry (No. 2016YFC0106204), the Shanghai Senior Integrative Chinese and Western Medicine Talents Program (No. ZY(20182020)-RCPY-2017) and the Pujiang Talent Project (No. 17PJ1407600). The funding facilitated the collection, analysis, and interpretation of the data and the writing of the manuscript.

\section{Availability of data and materials}

The datasets used and/or analyzed during the current study are available from the corresponding author on reasonable request.

Ethics approval and consent to participate

The project was approved by the Ethic Committee of Shanghai Jiao Tong University Affiliated Sixth People's Hospital. For this type of study

(retrospective chart review), formal consent is not required.

\section{Consent for publication}

Not Applicable.

Competing interests

The authors declare that they have no competing interests.

\section{Author details}

${ }^{1}$ Department of Oncology, Shanghai Jiao Tong University Affiliated Sixth People's Hospital, 600 Yishan Rd, Shanghai 200233, China. ²Department of Medical Oncology, Shanghai Jiao Tong University Affiliated Sixth People's Hospital South Campus, 6600 Nanfeng Rd, Shanghai 201499, China.

Received: 4 August 2019 Accepted: 27 November 2019

Published online: 02 December 2019

\section{References}

1. Robinson SI, Ahmed SK, Okuno SH, Arndt CA, Rose PS, Laack NN. Clinical outcomes of adult patients with relapsed Ewing sarcoma: a 30-year singleinstitution experience. Am J Clin Oncol. 2014;37(6):585-91.

2. Cotterill SJ, Ahrens S, Paulussen M, Jurgens HF, Voute PA, Gadner H, et al. Prognostic factors in Ewing's tumor of bone: analysis of 975 patients from the European intergroup cooperative Ewing's sarcoma study group. J Clin Oncol. 2000;18(17):3108-14.

3. Ganjoo KN, Patel S. The treatment outcome for adult patients with Ewing's sarcoma. Curr Oncol Rep. 2013;15(4):372-7.

4. Gaspar N, Hawkins DS, Dirksen U, Lewis IJ, Ferrari S, Le Deley MC, et al. Ewing sarcoma: current management and future approaches through collaboration. J Clin Oncol. 2015;33(27):3036-46.

5. Womer RB, West DC, Krailo MD, Dickman PS, Pawel BR, Grier HE, et al, Randomized controlled trial of interval-compressed chemotherapy for the treatment of localized Ewing sarcoma: a report from the Children's oncology group. J Clin Oncol. 2012;30(33):4148-54.

6. Grier HE, Krailo MD, Tarbell NJ, Link MP, Fryer CJ, Pritchard DJ, et al. Addition of ifosfamide and etoposide to standard chemotherapy for Ewing's sarcoma and primitive neuroectodermal tumor of bone. N Engl J Med. 2003;348(8): 694-701.

7. Burgert EO Jr, Nesbit ME, Garnsey LA, Gehan EA, Herrmann J, Vietti TJ, et al. Multimodal therapy for the management of nonpelvic, localized Ewing's sarcoma of bone: intergroup study IESS-II. J Clin Oncol. 1990;8(9):1514-24.

8. Juergens $C$, Weston C, Lewis I, Whelan J, Paulussen M, Oberlin O, et al. Safety assessment of intensive induction with vincristine, ifosfamide, doxorubicin, and etoposide (VIDE) in the treatment of Ewing tumors in the EURO-E.W.I.N.G. 99 clinical trial. Pediatr Blood Cancer. 2006;47(1):22-9.

9. Granowetter L, Womer R, Devidas M, Krailo M, Wang C, Bernstein M, et al. Dose-intensified compared with standard chemotherapy for nonmetastatic Ewing sarcoma family of tumors: a Children's oncology group study. J Clin Oncol. 2009;27(15):2536-41.

10. Wagner MJ, Livingston JA, Patel SR, Benjamin RS. Chemotherapy for bone sarcoma in adults. J Oncol Pract. 2016;12(3):208-16.

11. Bacci G, Ferrari S, Comandone A, Zanone A, Ruggieri P, Longhi A, et al. Neoadjuvant chemotherapy for Ewing's sarcoma of bone in patients older than thirty-nine years. Acta Oncol. 2000;39(1):111-6. 
12. Rodriguez-Galindo C, Liu T, Krasin MJ, Wu J, Billups CA, Daw NC, et al. Analysis of prognostic factors in Ewing sarcoma family of tumors: review of St. Jude Children's Research Hospital studies. Cancer. 2007;1 10(2):375-84.

13. Orr WS, Denbo JW, Billups CA, Wu J, Navid F, Rao BN, et al. Analysis of prognostic factors in extraosseous Ewing sarcoma family of tumors: review of St. Jude Children's Research Hospital experience. Ann Surg Oncol. 2012; 19(12):3816-22.

14. Paulussen M, Ahrens S, Dunst J, Winkelmann W, Exner GU, Kotz R, et al. Localized Ewing tumor of bone: final results of the cooperative Ewing's sarcoma study CESS 86. J Clin Oncol. 2001;19(6):1818-29.

15. Parkin DM, Stiller CA, Nectoux J. International variations in the incidence of childhood bone tumours. Int J Cancer. 1993;53(3):371-6.

16. Jawad MU, Cheung MC, Min ES, Schneiderbauer MM, Koniaris LG, Scully SP. Ewing sarcoma demonstrates racial disparities in incidence-related and sexrelated differences in outcome: an analysis of 1631 cases from the SEER database, 1973-2005. Cancer. 2009;115(15):3526-36.

17. Zhang J, Huang Y, Lu J, He A, Zhou Y, Hu H, et al. Impact of first-line treatment on outcomes of Ewing sarcoma of the spine. Am J Cancer Res. 2018:8(7):1262-72

18. Ladenstein R, Potschger U, Le Deley MC, Whelan J, Paulussen M, Oberlin O, et al. Primary disseminated multifocal Ewing sarcoma: results of the EuroEWING 99 trial. J Clin Oncol. 2010;28(20):3284-91.

19. Tao HT, Hu Y, Wang JL, Cheng Y, Zhang X, Wang H, et al. Extraskeletal Ewing sarcomas in late adolescence and adults: a study of 37 patients. Asian Pac J Cancer Prev. 2013;14(5):2967-71.

20. El Weshi A, Allam A, Ajarim D, Al Dayel F, Pant R, Bazarbashi S, et al. Extraskeletal Ewing's sarcoma family of tumours in adults: analysis of 57 patients from a single institution. Clin Oncol (R Coll Radiol). 2010;22(5): 374-81.

21. Ahmed SK, Robinson SI, Okuno SH, Rose PS, Issa Laack NN. Adult Ewing sarcoma: survival and local control outcomes in 36 patients with metastatic disease. Am J Clin Oncol. 2014;37(5):423-9.

22. Seker MM, Kos T, Ozdemir N, Seker A, Aksoy S, Uncu D, et al. Treatment and outcomes of Ewing sarcoma in Turkish adults: a single Centre experience. Asian Pac J Cancer Prev. 2014;15(1):327-30.

23. Gupta AA, Pappo A, Saunders N, Hopyan S, Ferguson P, Wunder J, et al. Clinical outcome of children and adults with localized Ewing sarcoma: impact of chemotherapy dose and timing of local therapy. Cancer. 2010; 116(13):3189-94

24. Casey DL, Meyers PA, Alektiar KM, Magnan H, Healey JH, Boland PJ, et al. Ewing sarcoma in adults treated with modern radiotherapy techniques. Radiother Oncol. 2014;113(2):248-53.

25. Lee J, Hoang BH, Ziogas A, Zell JA. Analysis of prognostic factors in Ewing sarcoma using a population-based cancer registry. Cancer. 2010;116(8): 1964-73.

26. Paioli A, Luksch R, Fagioli F, Tamburini A, Cesari M, Palmerini E, et al. Chemotherapy-related toxicity in patients with non-metastatic Ewing sarcoma: influence of sex and age. J Chemother. 2014;26(1):49-56.

27. Diaz-Beveridge R, Lorente D, Torres B, Canete A, Rodrigo E, Bruixola G, et al. Multimodality treatment of pediatric and adult patients with Ewing sarcoma: a single-institution experience. J Pediatr Hematol Oncol. 2015; 37(5):e278-84.

28. Biswas B, Bakhshi S. Management of Ewing sarcoma family of tumors: current scenario and unmet need. World J Orthop. 2016;7(9):527-38.

29. Verrill MW, Judson IR, Harmer CL, Fisher C, Thomas JM, Wiltshaw E. Ewing's sarcoma and primitive neuroectodermal tumor in adults: are they different from Ewing's sarcoma and primitive neuroectodermal tumor in children? J Clin Oncol. 1997;15(7):2611-21.

30. Fizazi K, Dohollou N, Blay JY, Guerin S, Le Cesne A, Andre F, et al. Ewing's family of tumors in adults: multivariate analysis of survival and long-term results of multimodality therapy in 182 patients. J Clin Oncol. 1998;16(12): 3736-43.

31. Lyman GH. Impact of chemotherapy dose intensity on cancer patient outcomes. J Natl Compr Cancer Netw. 2009;7(1):99-108.

32. Tahover E, Segal A, Isacson R, Rosengarten O, Grenader T, Gips M, et al. Dexrazoxane added to doxorubicin-based adjuvant chemotherapy of breast cancer: a retrospective cohort study with a comparative analysis of toxicity and survival. Anti-Cancer Drugs. 2017;28(7):787-94.

33. Reichardt P, Tabone MD, Mora J, Morland B, Jones RL. Risk-benefit of dexrazoxane for preventing anthracycline-related cardiotoxicity: reevaluating the European labeling. Future Oncol. 2018;14(25):2663-76.
34. Lipshultz SE, Scully RE, Lipsitz SR, Sallan SE, Silverman LB, Miller TL, et al. Assessment of dexrazoxane as a cardioprotectant in doxorubicin-treated children with high-risk acute lymphoblastic leukaemia: long-term follow-up of a prospective, randomised, multicentre trial. Lancet Oncol. 2010;11(10): 950-61.

35. Chow EJ, Asselin BL, Schwartz CL, Doody DR, Leisenring WM, Aggarwal S, et al. Late mortality after Dexrazoxane treatment: a report from the Children's oncology group. J Clin Oncol. 2015;33(24):2639-45.

\section{Publisher's Note}

Springer Nature remains neutral with regard to jurisdictional claims in published maps and institutional affiliations.

\section{Ready to submit your research? Choose BMC and benefit from:}

- fast, convenient online submission

- thorough peer review by experienced researchers in your field

- rapid publication on acceptance

- support for research data, including large and complex data types

- gold Open Access which fosters wider collaboration and increased citations

- maximum visibility for your research: over $100 \mathrm{M}$ website views per year

At BMC, research is always in progress.

Learn more biomedcentral.com/submissions 